\title{
Multibody Modelling of Wave Energy Converters Using Pseudo-Spectral Methods With Application to a Three-Body Hinge-Barge Device
}

\author{
Francesco Paparella, Giorgio Bacelli, Andrew Paulmeno, Sarah E. Mouring, \\ and John V. Ringwood, Senior Member, IEEE
}

\begin{abstract}
Multibody wave energy converters are composed of several bodies interconnected by joints. Two different formulations are adopted to describe the dynamics of multibody systems: the differential and algebraic equations (DAEs) formulation, and the ordinary differential equations (ODEs) formulation. While the number of variables required for the description of the dynamics of a multibody system is greater in the DAE formulation than in the ODE formulation, the ODE formulation involves an extra computational effort in order to describe the dynamics of the system with a smaller number of variables. In this paper, pseudospectral (PS) methods are applied in order to solve the dynamics of multibody wave energy converters using both DAE and ODE formulations. Apart from providing a solution to the dynamics of multibody systems, pseudo-spectral methods provide an accurate and efficient formulation for the control of multibody wave energy converters. As an application example, this paper focuses on the dynamic modeling of a three-body hinge-barge device, where wave-tank tests are carried out in order to validate the DAE and ODE models against experimental data. Comparison of the ODE and DAE PS methods against a reference model based on the straightforward (Runge-Kutta) integration of the equations of motion shows that pseudo-spectral methods are computationally more stable and require less computational effort for short time steps.
\end{abstract}

Index Terms-Multi-body wave energy converters, pseudospectral methods, model-based control.

\section{INTRODUCTION}

$\mathbf{M}$ ULTI-BODY wave energy converters are composed of an assembly of bodies connected together by different type of joints. The motion of each body is restrained by the kinematic constraints introduced by the joints. Two different formulations can be used to describe the dynamics of a multi-body system: the Differential and Algebraic Equations (DAEs) and the Ordinary Differential Equations

Manuscript received August 04, 2015; revised October 16, 2015; accepted December 05, 2015. This paper was supported by the Science Foundation Ireland under Grant 12/RC/2302 for the Marine Renewable Ireland (MaREI) Centre. Paper no. TSTE-00644-2015.

F. Paparella and J. V. Ringwood are with the Center for Ocean Energy Research (COER), National University of Ireland Maynooth, Co. Kildare, Ireland (e-mail: fpaparella@eeng.nuim.ie; john.ringwood@eeng.nuim.ie).

G. Bacelli is with Sandia National Laboratories, Albuquerque, NM 87185 USA (e-mail: gbacelli@sandia.gov).

A. Paulmeno and S. E. Mouring are with the Department of Naval Architecture and Ocean Engineering, U.S. Naval Academy, Annapolis, MD 21402 USA (e-mail: paulmenoa@yahoo.com; mouring@usna.edu).

Color versions of one or more of the figures in this paper are available online at http://ieeexplore.iee.org.

Digital Object Identifier 10.1109/TSTE.2015.2510699
(ODEs) formulation. In the DAE formulation, a set of redundant $n$ generalized coordinates is used to describe the dynamics of the system, and the equations of motion result in $2 n$ differential and $m$ constraint equations. In addition, $m$ unknowns called Lagrange multipliers are added into the differential equations. The Lagrange multipliers are algebraic variables, so that their time derivative does not feature in the equations. The resulting system is a set of DAEs for the generalized coordinates and Lagrange multipliers.

In the ODE formulation, the generalized coordinates are expressed with respect to a set of $(n-m)$ independent coordinates, also called degrees of the freedom, by means of the constraint equations. Therefore, the DAE system can be transformed into a reduced number of $2(n-m)$ ordinary differential equations (ODEs) for the independent coordinates with elimination of the Lagrange multipliers.

Regarding solution techniques for DAE systems, index reduction techniques combined with backwardward difference methods (BDFs) have been proposed in [1]. The index of a DAE system composed of the Euler-Lagrange equations is reduced from three to two, and then a variable-order, variable-step BDF method is applied to the resulting system of equations.

Alternatively, the DAE system can be reduced to an ODE system by means of an appropriate transformation of coordinates. In Maggi's formulation [2], the generalized velocities are expressed in terms of the kinematic characteristics, which are the velocities of the degrees of freedom. Then, the EulerLagrange equations are derived for the kinematic characteristics with elimination of the Lagrange multipliers by means of the null-space of the constraint matrix [3], [4]. In the Index-1 formulation [5], the Euler-Lagrange equations for the generalized coordinates, together with the constraints at the acceleration level, form an Index-1 system of DAEs which can be solved for the generalized coordinates and Lagrange multipliers. Then, the accelerations are integrated in order to obtain the positions and velocities of the generalized coordinates. In the Udwadia and Kalabas Formulation (UKF) [6], a more compact form of the Index-1 formulation was derived by means of the Moore-Penrose Generalized Inverse (MPGI). In the Null Space Formulation (NSF) [5], [7], [8], the Lagrange multipliers that appear in the Index 1 formulation are eliminated from the Euler-Lagrange equations by means of the null space introduced in Maggi's formulation. Therefore, a system of second order ODEs for the generalized coordinates is obtained. 
The number of variables needed to describe the dynamics of a constrained system in the DAE formulation is $2 n+$ $m$ ( $n$ generalized positions, $n$ generalized velocities and $m$ Lagrange's multipliers), while for the ODE formulation, the number of variables is reduced to $2 n$ for the Index- 1 formulation, NSF and UKF, and $2(n-m)$ for Maggi's formulation. However, a reduction in the number of variables requires an extra computational effort; in Maggi's and the NSF formulation, the computation of the null space of the constraint matrix is required, while in the UKF formulation, MPGIs are calculated. Nevertheless, the matrices in the DAE formulation are characterized by a high sparsity, so that efficient solution techniques can be used [9], [10].

In this paper, pseudo-spectral (PS) methods are applied to both the DAE and ODE formulations, in order to obtain the solution for the dynamics of a multi-body system. PS methods are a subset of the class of techniques used for the discretisation of integral and partial differential equations known as mean weighted residuals [11], [12]. Apart from providing a solution for the dynamics of a multi-body system, PS methods can also be used to efficiently solve an optimal control problem for the device [13].

The remainder of the paper is organized as follows: in Section (II), the DAE and ODE formulations are applied to a multi-body system, while, in Section III, PS methods are used to obtain a solution for the dynamics of a multi-body system. In Section IV, a three-body hinge-barge device is considered as a case study, and the DAE and ODE formulations are applied in order to derive the equations of motion. Finally, in Section V, the DAE and ODE formulations applied to the device are compared against tank test data to verify their validity. Computational aspects are considered in Section VI, while overall conclusions are drawn in Section VII.

\section{EQuATions of Motion of A Multi-Body System}

\section{A. Reference Frames}

For the description of the motion of a body in space, six coordinates are required: three coordinates for position and three coordinates for rotation. For the analysis of multi-body systems, two types of coordinate frames are defined: the global or inertial frame and the body frame. The global frame is fixed to a point in space, and therefore its position and orientation are constant in time. The body frame is attached to a point of the body, and its position and orientation change with time. A body frame is assigned to each body composing a multi-body system. In Figure 1, a free floating unconstrained body $k$ is represented together with a global frame $X_{i} Y_{i} Z_{i}$ and a body frame $X_{b} Y_{b} Z_{b}$. The vector $\mathbf{p}_{i, b_{k}} \in \mathbb{R}^{3 \times 1}$ represents the position of the origin of the body frame $O_{b_{k}}$ with respect to the point $i$. The components of the vector $\mathbf{p}_{i, b_{k}}$ can be expressed from the body frame to the global frame by using the following transformation:

$$
\mathbf{p}_{i, b_{k}}^{i}=\mathbf{R}_{b}^{i}\left(\Theta_{k}\right) \mathbf{p}_{i, b_{k}}^{b}
$$

where the vectors $\mathbf{p}_{i, b_{k}}^{b}$ and $\mathbf{p}_{i, b_{k}}^{i}$ represent the position vector of $O_{b_{k}}$ with respect to the point $i$, expressed in the body frame and global frame, respectively. The matrix $\mathbf{R}_{b}^{i}\left(\boldsymbol{\Theta}_{k}\right) \in \mathbb{R}^{3 \times 3}$, used for the transformation of coordinates, is a function of the

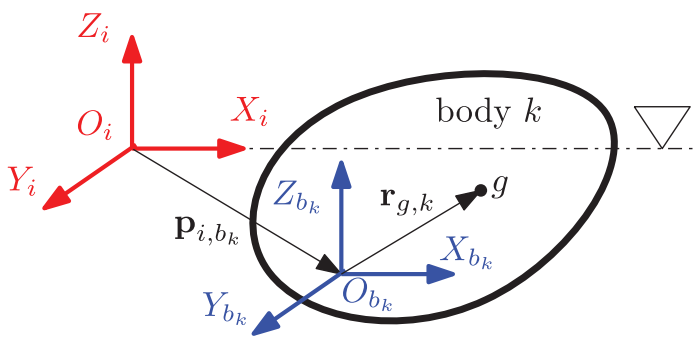

Fig. 1. A free floating unconstrained body $k$, where $X_{i} Y_{i} Z_{i}$ and $X_{b} Y_{b} Z_{b}$ represent the global and body reference frames, respectively.

vector of Euler angles $\boldsymbol{\Theta}_{k}=[\phi \theta \psi]^{T}$, where $\phi$ is the roll angle, $\theta$ the pitch angle and $\psi$ the yaw angle of the body [14]. The velocities of the Euler angles can be obtained from the angular velocity vector $\boldsymbol{\omega}_{i, b}^{b}$ expressed in the body frame coordinates as follows:

$$
\dot{\boldsymbol{\Theta}}_{k}=\mathbf{T}\left(\Theta_{k}\right) \boldsymbol{\omega}_{i, b_{k}}^{b}
$$

where the matrix $\mathbf{T}\left(\boldsymbol{\Theta}_{k}\right)$ is a function of Euler angles, and can be obtained by linear superposition of the rotations of the body frame around its axis [14].

\section{B. Dynamics of an Unconstrained Body}

The Newton-Euler equations of motion for a free floating unconstrained body are represented as a system of first-order integro-differential equations as follows [14]:

$$
\begin{aligned}
& \dot{\mathbf{q}}_{k}=\mathbf{J}^{b_{k}}(\boldsymbol{\Theta}) \mathbf{v}_{k} \\
& \mathbf{M}^{b_{k}} \dot{\mathbf{v}}_{k}+\left(\mathbf{B}^{b_{k}}+\mathbf{B}_{v i s c}^{b_{k}}\right) \mathbf{v}_{k}=-\mathbf{G}^{b_{k}} \boldsymbol{q}_{k}-\mathbf{M}_{\infty}^{b_{k}} \dot{\mathbf{v}}_{k}+. . \\
& \quad-\int_{-\infty}^{t} \mathbf{K}_{\text {rad }}^{b_{k}}(t-\tau) \mathbf{v}_{k}, d \tau+\mathbf{f}_{\text {wave }}^{b_{k}}+\mathbf{f}_{\text {ext }}^{b_{k}}
\end{aligned}
$$

where:

$$
\begin{aligned}
& \mathbf{q}_{k}=\left[\begin{array}{ll}
\mathbf{p}^{i, b_{k}}{ }_{i, \Theta_{k}}^{T} & \boldsymbol{\Theta}_{k}^{T}
\end{array}\right]^{T} \\
& \mathbf{v}_{k}=\left[\begin{array}{ll}
\mathbf{v}^{b^{T}}{ }_{i, b_{k}} & \boldsymbol{\omega}^{b^{T} b_{k}}
\end{array}\right]^{T} \\
& \mathbf{J}^{b_{k}}(\boldsymbol{\Theta})=\left[\begin{array}{cc}
\mathbf{R}_{b}^{i}\left(\boldsymbol{\Theta}_{k}\right) & \mathbf{0}_{3 \times 3} \\
\mathbf{0}_{3 \times 3} & \mathbf{T}\left(\boldsymbol{\Theta}_{k}\right)
\end{array}\right] \\
& \mathbf{M}^{b_{k}}=\left[\begin{array}{cc}
m_{k} \mathbf{I}_{3 \times 3} & -m_{k} \mathbf{S}\left(\mathbf{r}_{g, k}\right) \\
m_{k} \mathbf{S}\left(\mathbf{r}_{g, k}\right) & \mathbf{I}_{b_{k}}
\end{array}\right] \\
& \mathbf{B}^{b_{k}}=\left[\begin{array}{cc}
m_{k} \mathbf{S}\left(\boldsymbol{\omega}_{i, b_{k}}^{b}\right) & -m_{k} \mathbf{S}\left(\boldsymbol{\omega}_{i, b_{k}}^{b}\right) \mathbf{S}\left(\mathbf{r}_{g, k}\right) \\
m_{k} \mathbf{S}\left(\mathbf{r}_{g, k}\right) \mathbf{S}\left(\boldsymbol{\omega}_{i, b_{k}}^{b}\right) & -\mathbf{S}\left(\mathbf{I}_{b_{k}} \boldsymbol{\omega}_{i, b_{k}}^{b}\right)
\end{array}\right] \\
& \mathbf{f}_{\text {wave }}^{b_{k}}=\int_{-\infty}^{\mathbf{K}_{\text {wave }}^{b_{k}}(t-\tau) \eta(\tau), d \tau} \\
& \mathbf{f}_{\text {ext }}^{b_{k}}=\mathbf{f}_{P T O}^{b_{k}}+\mathbf{f}^{b_{k}}
\end{aligned}
$$

with $m_{k}$ the mass of the body, $\mathbf{r}_{g, k}$ the distance vector of the center of mass from the origin of the body frame, $\mathbf{I}_{b_{k}}$ the inertia matrix of the body around the origin of the body frame, $\mathbf{M}^{b_{k}}$ the rigid-body inertia matrix of the body, $\mathbf{B}^{b_{k}}$ the CoriolisCentripetal matrix, $\mathbf{B}_{\text {visc }}^{b_{k}}$ the linearized viscous damping, $\mathbf{G}^{b_{k}}$ the hydrostatic matrix, $\mathbf{f}_{\text {wave }}^{b_{k}}$ the vector of the excitation forces due the action of the waves on the body, $\mathbf{f}_{P T O}^{b_{k}}$ the force vector due to the Power Take-Off (PTO) system, $\mathbf{f}^{b_{k}}$ the vector 
of forces due to moorings, hinge-friction, etc. , $\mathbf{K}_{r a d}^{b_{k}}$ contains impulse response functions of the radiation forces, $\mathbf{K}_{\text {wave }}^{b_{k}}$ contains the impulse response functions of the wave excitation forces, $\mathbf{M}_{\infty}^{b_{k}}$ the added mass at infinite frequency, $\eta$ the free surface elevation and $\mathbf{S}$ a skew-symmetric matrix for the cross-product $\mathbf{a} \times \mathbf{b}:=\mathbf{S}(\mathbf{a}) \mathbf{b}$.

The terms on the left-hand side of equation (4) represent the rigid-body dynamics of the unconstrained body, expressed about the origin of the body frame $X_{b} Y_{b} Z_{b}$. The hydrodynamic parameters $\mathbf{G}^{b_{k}}, \mathbf{M}_{\infty}^{b_{k}}, \mathbf{K}_{\text {rad }}^{b_{k}}$ and $\mathbf{K}_{\text {wave }}^{b_{k}}$ are computed by means of the boundary element software WAMIT [15], which computes all the quantities in the hydrodynamic $h$-frame. The transformation matrix $\mathbf{R}_{b}^{h}$ is used to convert the hydrodynamic parameters from the $h$-frame to the body frame [16]. Under the assumption of small oscillations of the body frame with respect to the $h$-frame, the matrix $\mathbf{R}_{b}^{h}$ reduces to the identity matrix $\mathbf{I}_{3 \times 3}$.

\section{DAE Formulation for $N$ Interconnected Bodies}

In case of $N$ interconnected bodies, $m$ algebraic equations are required in order to describe the constraints introduced by the joints. The constraint equations can be represented as follows:

$$
\mathbf{C}(\mathbf{z}, t)=0
$$

where $\mathbf{z}=\left[\begin{array}{lll}\mathbf{z}_{1}^{T} & . . & \mathbf{z}_{N}^{T}\end{array}\right]^{T} \in \mathbb{R}^{(6 \times N) \times 1}$ and $\mathbf{z}_{k}=\left[\mathbf{p}^{b^{T}}{ }_{i, b_{k}} \Theta_{k}^{T}\right]^{T}$, with $k=1, . . N$. The constraint equations (12) are considered to be holonomic, since the generalized velocities do not appear in the equations. An additional term, representing the constraint forces, is added into the Newton-Euler equations of motion. The constraint forces are represented by a set of $m$ Lagrange multipliers $\lambda$, which are algebraic variables since their time derivatives do not appear in the equations of motion. Thus, the equations of motion for a system composed of $N$ interconnected bodies can be represented by a set of DAEs, given as follows [17]:

$$
\begin{aligned}
& \dot{\mathbf{q}}=\mathbf{J}(\boldsymbol{\Theta}) \mathbf{v} \\
& \mathbf{M} \dot{\mathbf{v}}+\left(\mathbf{B}+\mathbf{B}_{v i s c}\right) \mathbf{v}+\mathbf{C}_{z}^{T} \boldsymbol{\lambda}=-\mathbf{G q}-\mathbf{M}_{\infty} \dot{\mathbf{v}}+. . \\
& \quad-\int_{-\infty}^{t} \mathbf{K}_{\text {rad }}(t-\tau) \mathbf{v}, d \tau+\mathbf{f}_{\text {wave }}+\mathbf{f}_{e x t} \\
& \mathbf{C}(\mathbf{z}, t)=0
\end{aligned}
$$

where:

$$
\begin{aligned}
& \mathbf{q}=\left[\begin{array}{lll}
\mathbf{q}_{1}^{T} & \mathbf{q}_{2}^{T} \ldots \mathbf{q}_{N}^{T}
\end{array}\right]^{T} \\
& \mathbf{v}=\left[\begin{array}{lll}
\mathbf{v}_{1}^{T} & \mathbf{v}_{2}^{T} & . . \mathbf{v}_{N}^{T}
\end{array}\right]^{T} \\
& \mathbf{J}(\boldsymbol{\Theta})=\operatorname{diag}\left(\mathbf{J}^{b_{1}}(\mathbf{\Theta}), . ., \mathbf{J}^{b_{N}}(\boldsymbol{\Theta})\right. \\
& \mathbf{M}=\operatorname{diag}\left(\mathbf{M}^{b_{1}}, . ., \mathbf{M}^{b_{N}}\right) \\
& \mathbf{B}=\operatorname{diag}\left(\mathbf{B}^{b_{1}}, . ., \mathbf{B}^{b_{N}}\right) \\
& \mathbf{B}_{v i s c}=\operatorname{diag}\left(\mathbf{B}_{v i s c}^{b_{1}}, . ., \mathbf{B}_{v i s c}^{b_{N}}\right) \\
& \mathbf{G}=\operatorname{diag}\left(\mathbf{G}^{b_{1}}, . ., \mathbf{G}^{b_{1}}\right) \\
& \mathbf{M}_{\infty}=\operatorname{diag}\left(\mathbf{M}_{\infty}^{b_{1}}, . ., \mathbf{M}_{\infty}^{b_{N}}\right)
\end{aligned}
$$

$$
\begin{aligned}
& \mathbf{f}_{\text {wave }}=\left[\mathbf{f}_{\text {wave }}^{b_{\text {wave }}{ }^{T}} \mathbf{f}_{\text {wave }}^{b_{N}{ }^{T}}\right]^{T} \\
& \mathbf{f}_{e x t}=\left[\mathbf{f}_{e x t}^{b_{1}{ }^{T}} \ldots \mathbf{f}_{e x t}^{b_{N}{ }^{T}}\right]^{T}
\end{aligned}
$$

The matrix $\mathbf{C}_{z}$ represents the partial derivative of the constraint equations with respect to the vector of generalized positions z. In the DAE formulation, the total number of variables required to describe the motion of $N$ interconnected bodies is $2 \times(6 \times N)+m$, i.e. $6 \times N$ positions, $6 \times N$ velocities and $m$ Lagranges multipliers.

\section{ODE Formulation for $N$ Interconnected Bodies}

As an alternative to the DAE formulation, using the constraints in equation (12), the dynamics of $N$ interconnected bodies can be described by means of a set of $n=(6 \times N-m)$ independent coordinates, or degrees of freedom (DoF). The vector of generalized velocities $\mathbf{v}$ can be partitioned into dependent velocities $\mathbf{v}_{d}$, and independent velocities $\mathbf{v}_{s}$, as follows:

$$
\mathbf{v}=\left[\begin{array}{c}
\mathbf{v}_{d} \\
\mathbf{v}_{s}
\end{array}\right]
$$

$\mathbf{v}_{d}$ and $\mathbf{v}_{s}$ are related as follows [17]:

$$
\mathbf{v}_{d}=\mathbf{C}_{s} \mathbf{v}_{s}=-\mathbf{C}_{\mathbf{q}_{d}}^{-1} \mathbf{C}_{\mathbf{q}_{s}} \mathbf{v}_{s}
$$

where $\mathbf{C}_{\mathbf{q}_{d}}$ and $\mathbf{C}_{\mathbf{q}_{s}}$ represent the partial derivatives of the constraint equations associated with the dependent and independent coordinates, respectively. Therefore, the vector of generalized velocities $\mathbf{v}$ can be written in terms of the independent velocities $\mathbf{v}_{s}$, as follows:

$$
\mathbf{v}=\mathbf{P v}_{s}=\left[\begin{array}{l}
\mathbf{C}_{s} \\
\mathbf{I}_{n}
\end{array}\right] \mathbf{v}_{s}
$$

where $\mathbf{I}_{n}$ is the identity matrix of dimension $n$. The time derivative of the generalized velocities can be expressed as:

$$
\dot{\mathbf{v}}=\mathbf{P} \dot{\mathbf{v}}_{s}+\dot{\mathbf{P}}_{s}
$$

Substituting (28) and (29) into (14), and multiplying both sides of the resulting equation by $\mathbf{P}^{T}$, the following system of first order integro-differential equations is obtained:

$$
\begin{aligned}
& \dot{\mathbf{q}}_{s}=\mathbf{v}_{s} \\
& \mathbf{M}_{s} \dot{\mathbf{v}}_{s}+\left(\mathbf{B}_{s}+\mathbf{B}_{v i s c, s}\right) \mathbf{v}_{s}=-\mathbf{G}_{s} \mathbf{q}_{s}-\mathbf{M}_{\infty, s} \dot{\mathbf{v}}_{s}+. . \\
& \quad-\int_{-\infty}^{t} \mathbf{K}_{\text {rad,s }}(t-\tau) \mathbf{v}_{s}, d \tau+\mathbf{f}_{\text {wave }, s}+\mathbf{f}_{e x t, s}
\end{aligned}
$$

where:

$$
\begin{aligned}
& \mathbf{M}_{s}=\mathbf{P}^{T} \mathbf{M P} \\
& \mathbf{B}_{s}=\mathbf{P}^{T} \mathbf{B P}+\mathbf{P}^{T} \mathbf{M} \dot{\mathbf{P}}+\mathbf{P}^{T} \mathbf{M}_{\infty} \dot{\mathbf{P}} \\
& \mathbf{B}_{\text {visc }, s}=\mathbf{P}^{T} \mathbf{B}_{\text {visc }} \mathbf{P} \\
& \mathbf{G}_{s}=\mathbf{P}^{T} \mathbf{G P} \\
& \mathbf{M}_{\infty, s}=\mathbf{P}^{T} \mathbf{M}_{\infty} \mathbf{P} \\
& \mathbf{K}_{\text {rad }, s}=\mathbf{P}^{T} \mathbf{K}_{\text {rad }} \mathbf{P} \\
& \mathbf{f}_{\text {wave }, s}=\mathbf{P}^{T} \mathbf{f}_{\text {wave }} \\
& \mathbf{f}_{\text {ext }, s}=\mathbf{P}^{T} \mathbf{f}_{\text {ext }}
\end{aligned}
$$


Thus, the equations of motion of a system composed by $N$ interconnected bodies can be represented by a set of ODEs for the independent coordinates. In the ODE formulation, the total number of variables required for describing the motion is $2 n$ ( $n$ positions and $n$ velocities).

\section{Pseudo-Spectral Approximation Methods}

In this section, PS methods are used to compute an approximate solution to the integro-differential equations obtained for the DAE and ODE formulations. The positions and velocities that appear in the equations of motion obtained for the DAE and ODE formulations can be approximated with a linear combination of basis functions. Given the periodic nature of the variables associated with the problem, non-zero mean trigonometric polynomials (truncated Fourier series) represent a sensible choice for the approximation of positions and velocities. Therefore, the $i$ th components of the position and velocity vector are given as follows:

$$
\begin{aligned}
q_{i}(t) \approx q_{i}^{N_{x}}(t) & =\sum_{k=0}^{N_{x}} x_{i, k}^{q, c} \cos \left(k \omega_{0} t\right)+x_{i, k}^{q, s} \sin \left(k \omega_{0} t\right) \\
& =\boldsymbol{\Phi}(t) \hat{\mathbf{x}}_{i}^{q} \\
v_{i}(t) \approx v_{i}^{N_{x}}(t) & =\sum_{k=0}^{N_{x}} x_{i, k}^{v, c} \cos \left(k \omega_{0} t\right)+x_{i, k}^{v, s} \sin \left(k \omega_{0} t\right) \\
& =\boldsymbol{\Phi}(t) \hat{\mathbf{x}}_{i}^{v}
\end{aligned}
$$

where $i=1, . ., 6 N$ and $i=1, . ., n$ for the DAE and ODE formulations, respectively. The parameter $N_{x}$ is the order of expansion for the position and velocity of the states. The vector of the coefficients $\hat{\mathbf{x}}_{i}^{q}$ and $\hat{\mathbf{x}}_{i}^{v}$ of the approximated $i$ th components of the position and velocity vector are given as follows:

$$
\begin{aligned}
\hat{\mathbf{x}}_{i}^{q} & =\left[\begin{array}{lllll}
x_{i, 1}^{q, c} & x_{i, 1}^{q, s} & \ldots & x_{i, N_{x}}^{q, c} & x_{i, N_{x}}^{q, s}
\end{array}\right]^{T} \\
\hat{\mathbf{x}}_{i}^{v} & =\left[\begin{array}{lllll}
x_{i, 1}^{v, c} & x_{i, 1}^{v, s} & \ldots & x_{i, N_{x}}^{v, c} & x_{i, N_{x}}^{v, s}
\end{array}\right]^{T}
\end{aligned}
$$

and the vector of the basis function $\mathbf{\Phi}(t)$ is given as follows:

$$
\boldsymbol{\Phi}(t)=\left[\cos \left(\omega_{0} t\right) \sin \left(\omega_{0} t\right) \ldots \cos \left(N_{x} \omega_{0} t\right) \sin \left(N_{x} \omega_{0} t\right)\right]^{T}
$$

where $\omega_{0}=2 \pi / T$ is the fundamental frequency. The derivatives of the $i$ th components of the position and velocity vector are, respectively,

$$
\begin{aligned}
& \dot{q}_{i}^{N_{x}}(t)=\dot{\boldsymbol{\Phi}}(t)^{T} \hat{\mathbf{x}}_{i}^{q}=\boldsymbol{\Phi}(t)^{T} \mathbf{D}_{\phi} \hat{\mathbf{x}}_{i}^{q} \\
& \dot{v}_{i}^{N_{x}}(t)=\dot{\boldsymbol{\Phi}}(t)^{T} \hat{\mathbf{x}}_{i}^{v}=\boldsymbol{\Phi}(t)^{T} \mathbf{D}_{\phi} \hat{\mathbf{x}}_{i}^{v}
\end{aligned}
$$

where $\mathbf{D}_{\phi} \in \mathbb{R}^{2 N_{x} \times 2 N_{x}}$ is a block diagonal matrix, with the $k$-th block is given as follows:

$$
\mathbf{D}_{\phi, k}=\left[\begin{array}{cc}
0 & k \omega_{0} \\
-k \omega_{0} & 0
\end{array}\right]
$$

Regarding the DAE formulation, substituting the approximated states (40), (41) and their time derivatives (45), (46) into the equations of motion (13)-(15), yields the following equations of motion in residual form:

$$
\begin{aligned}
r_{i}^{q}(t)= & \boldsymbol{\Phi}(t) \mathbf{D}_{\phi} \widehat{\mathbf{x}}_{i}^{q}-\sum_{p=1}^{6 N} J_{i, p} \boldsymbol{\Phi}(t) \widehat{\mathbf{x}}_{p}^{v} \\
r_{i}^{v}(t)= & \sum_{p=1}^{6 N} M_{i, p} \boldsymbol{\Phi}(t) \mathbf{D}_{\phi} \widehat{\mathbf{x}}_{p}^{v}+\sum_{p=1}^{6 N} B_{i, p} \boldsymbol{\Phi}(t) \widehat{\mathbf{x}}_{p}^{v} \\
& +\sum_{p=1}^{6 N} G_{i, p} \boldsymbol{\Phi}(t) \widehat{\mathbf{x}}_{p}^{q}+\sum_{p=1}^{6 N} \int_{-\infty}^{t} K_{\operatorname{rad}_{i, p}}(t-\tau) \boldsymbol{\Phi}(\tau) \widehat{\mathbf{x}}_{p}^{v} d \tau \\
& +\sum_{p=1}^{m} C_{q_{i, p}}^{T} \boldsymbol{\Phi}(t) \widehat{\mathbf{x}}_{p}^{\lambda}(t)-f_{\text {wave }, i}(t)-f_{i}(t) \\
r_{j}^{C}(t)= & C_{j}(\mathbf{q}, t)
\end{aligned}
$$

where $i=1, . ., 6 N, j=1, . ., m$, and $J_{i, p}, M_{i, p}, B_{i, p}, G_{i, p}$, $K_{\text {radi,p }_{i, p}}$ and $C_{q_{i, p p}}^{T}$ are the elements of the matrix $\mathbf{J}(\boldsymbol{\Theta}), \mathbf{M}$, $\mathbf{B}, \mathbf{G}, \mathbf{K}_{\text {rad }}$ and $\mathbf{C}_{q}^{T}$, respectively. Regarding the ODE formulation, substituting the approximated states (40), (41) and their time derivatives (45), (46) into the equations of motion (30)-(31), yields the following equations of motion in residual form:

$$
\begin{aligned}
& r_{i}^{q}(t)=\boldsymbol{\Phi}(t) \mathbf{D}_{\phi} \hat{\mathbf{x}}_{i}^{q}-\mathbf{\Phi}(t) \hat{\mathbf{x}}_{i}^{v} \\
& r_{i}^{v}(t)=\sum_{p=1}^{n} M_{s_{i, p}} \boldsymbol{\Phi}(t) \mathbf{D}_{\phi} \hat{\mathbf{x}}_{p}^{v}(t)+\sum_{p=1}^{n} B_{s_{i, p}} \boldsymbol{\Phi}(t) \hat{\mathbf{x}}_{p}^{v} \\
& \quad+\sum_{p=1}^{n} G_{s_{i, p}} \boldsymbol{\Phi}(t) \hat{\mathbf{x}}_{p}^{q}+\sum_{p=1}^{n} \int_{-\infty}^{t} K_{\text {rad } s_{i, p}}(t-\tau) \boldsymbol{\Phi}(\tau) \hat{\mathbf{x}}_{p}^{v} d \tau \\
& \quad-f_{\text {wave } s_{i}}(t)-f_{s_{i}}(t)
\end{aligned}
$$

where $i=1, . ., n$, and $M_{s_{i, p}}, B_{s_{i, p}}, G_{s_{i, p}}$, and $K_{r a d, s_{i, p}}$ are the elements of the matrix $\mathbf{M}_{s}, \mathbf{B}_{s}, \mathbf{G}_{s}$ and $\mathbf{K}_{r a d, s}$, respectively. PS methods are used to compute the coefficients $\hat{\mathbf{x}}_{i}^{q}$ and $\hat{\mathbf{x}}_{i}^{v}$ that minimize the residuals (48)-(50) and (51)-(52) for the DAE and ODE formulations, respectively [18]. The PS methods force the residuals of the equations of motion to be zero at a certain number of points in time $t_{k}$, called nodes. If the number of nodes is $N_{c}$, then a nonlinear system of $(2 \times 6 N+m) \times N_{c}$ and $2 \times n \times N_{c}$ equations is solved for the DAE and ODE formulations, respectively. The number of nodes depends on multiple factors, including the order of the expansion $N_{x}$ [19].

\section{Case Study: Three-Body Hinge-Barge Device}

A three-body hinge-barge device is considered as a case study. As shown in Figure 2, a hinge-barge device is composed of a number of rectangular bodies interconnected by hinges. The hinge-barge is an attenuator device since it operates along the wave direction. Examples of such systems include the McCabe Wave Pump (MWP) [20] and the SeaPower Platform [21]. Generally, the relative pitch motion between bodies is used to drive a Power TakeOff (PTO) system in order to convert the energy from the waves into mechanical energy. The damping plate connected to body 2 aims to reduce the vertical motion of body 2 , increasing the pitch motions of body 1 and 
3. The analysis of the motion of the devices is restricted to the two dimensional plane $X-Z$. In Figure 2, the device is represented together with the global frame $X_{g} Z_{g}$, while a body frame is assigned to each body composing the device. The total number of degrees of freedom of the system in Figure 2is four: the heave displacement $z_{2}$ of body 2 , and the pitch angles $\theta_{1}$, $\theta_{2}$ and $\theta_{3}$ of bodies 1,2 and 3 , respectively.

\section{A. DAE Formulation for a Three-Body Hinge-Barge Device}

In the following subsection, the DAE formulation is applied in order to obtain the equations of motion for a three-body hinge-barge device. The vector of generalized positions considered for the three-body hinge-barge device is:

$$
\mathbf{z}=\left[\begin{array}{lll}
\mathbf{z}_{1} & \mathbf{z}_{2} & \mathbf{z}_{3}
\end{array}\right]^{T}=\left[\begin{array}{llllll}
z_{i, b_{1}}^{b} & \theta_{1} & z_{i, b_{2}}^{b} & \theta_{2} & z_{i, b_{3}}^{b} & \theta_{3}
\end{array}\right]^{T}
$$

where $z_{i, b_{k}}^{b}$ and $\theta_{k}$ are the heave displacement and pitch angle of body $k$, respectively, with $k=1,2,3$. The block matrix $\mathbf{J}_{k}(\boldsymbol{\Theta})$ of the transformation matrix $\mathbf{J}(\boldsymbol{\Theta})$ in equation (18) is given as:

$$
\mathbf{J}^{b_{k}}=\left[\begin{array}{cc}
c\left(\theta_{k}\right) & 0 \\
0 & 1
\end{array}\right]
$$

where $k=1,2,3$. The block matrix $\mathbf{M}_{k}$ of the rigid-body inertia matrix $\mathbf{M}$ in equation (19) is given as:

$$
\mathbf{M}^{b_{k}}=\left[\begin{array}{cc}
m_{k} & 0 \\
0 & I_{y y, k}+m_{k} h_{g, k}^{2}
\end{array}\right]
$$

where $k=1,2,3, m_{k}$ is the mass of body $k, I_{y y, k}$ is the moment of inertia of body $k$ around the $y$-axis and $h_{g, k}$ is the distance of the center of mass of body $k$ from point $O_{b k}$ along the $z$-axis. The hydrodynamic loads $\mathbf{G}, \mathbf{M}_{\infty}, \mathbf{K}_{\text {rad }}$ and $\mathbf{f}_{\text {wave }}$ in equations (22), (23), (14) and (24) respectively, are obtained by means of the boundary element software WAMIT [15]. The vector of external forces $\mathbf{f}_{e x t}$ in equation (25) is given by the forces due to the PTO systems connecting body 2 to bodies 1 and 3. As shown in Figure 2, each PTO can be modeled as a linear dash-pot system. Considering the PTO connecting points $p_{1}$ and $p_{2}$, the axial force is given as follows:

$$
F_{s 1}=c_{1} \dot{l}_{1}
$$

where $c_{1}$ and $l_{1}$ are the damping coefficient and length of the dash-pot system, respectively. For small displacements of body 1 and $2, i_{1}$ can be calculated as follows:

$$
\dot{l}_{1}=a\left(\dot{\theta}_{2}-\dot{\theta}_{1}\right)
$$

where $a$ represent the vertical distance between the line of action of the PTO force and the center-line of the device. Similarly, for the PTO connecting points $p_{3}$ and $p_{4}$, the axial force is given as follows:

$$
F_{s 2}=c_{2} \dot{l}_{2}
$$

with $\dot{l}_{2}$ calculated as follows:

$$
\dot{l}_{2}=a\left(\dot{\theta}_{3}-\dot{\theta}_{2}\right)
$$

Therefore, the vector of loads, due to the PTO systems acting on the device, is given as follows:

$$
\mathbf{f}_{P T O}=-\left[\begin{array}{c}
0 \\
-F_{s 1} a \\
0 \\
F_{s 1} a-F_{s 2} a \\
0 \\
F_{s 2} a
\end{array}\right]
$$

Now, the constraint equations for the three-body hinge-barge device are derived. The hinge between body 2 and 3 introduces the following constraint equations:

$$
\mathbf{R}_{b}^{i}\left(\theta_{2}\right)\left(\left[\begin{array}{c}
d_{2} \\
z_{i, b_{2}}^{b}
\end{array}\right]+\left[\begin{array}{c}
-l_{3} \\
0
\end{array}\right]\right)-\mathbf{R}_{b}^{i}\left(\theta_{3}\right)\left(\left[\begin{array}{c}
d_{3} \\
z_{i, b 3}^{b}
\end{array}\right]+\left[\begin{array}{c}
l_{4} \\
0
\end{array}\right]\right)=0
$$

where $l_{3}$ and $l_{4}$ are the distances of the hinge from $O_{b_{2}}$ and $O_{b_{3}}$, respectively, while $d_{2}$ and $d_{3}$ are the distances of $O_{b_{2}}$ and $O_{b_{3}}$ from the global frame $X_{g} Z_{g}$, respectively. The hinge between body 1 and 2 introduces the following constraint equations:

$$
\mathbf{R}_{b}^{i}\left(\theta_{1}\right)\left(\left[\begin{array}{c}
d_{1} \\
z_{i, b_{1}}^{b}
\end{array}\right]+\left[\begin{array}{c}
-l_{1} \\
0
\end{array}\right]\right)-\mathbf{R}_{b}^{i}\left(\theta_{2}\right)\left(\left[\begin{array}{c}
d_{2} \\
z_{i, b 2}^{b}
\end{array}\right]+\left[\begin{array}{c}
l_{2} \\
0
\end{array}\right]\right)=0
$$

where $l_{1}$ and $l_{2}$ are the distances of the hinge from $O_{b_{1}}$ and $O_{b_{2}}$, respectively, while $d_{1}$ is the distance of $O_{b_{1}}$ from the global frame $X_{g} Z_{g}$. The rotation matrices $\mathbf{R}_{b}^{i}$ are given as follows:

$$
\mathbf{R}_{b}^{i}\left(\theta_{k}\right)=\left[\begin{array}{cc}
c\left(\theta_{k}\right) & -s\left(\theta_{k}\right) \\
s\left(\theta_{k}\right) & c\left(\theta_{k}\right)
\end{array}\right]
$$

where $c\left(\theta_{k}\right)=\cos \left(\theta_{k}\right)$ and $s\left(\theta_{k}\right)=\sin \left(\theta_{k}\right)$, with $k=1,2,3$. The constraints in equation (61) force the global position of the hinge defined by the coordinates of body 2 to be equal to the global position of the hinge defined by the coordinates of body 3. Similar considerations can be made for the constraints in equation (62). The matrix of the partial derivatives of constraint equations (61) and (62) computed with respect to the generalized positions and linearized around the equilibrium position, is given as follows:

$$
\mathbf{C}_{z}=\left[\begin{array}{cccccc}
0 & 0 & 1 & -l_{3} & -1 & -l_{4} \\
1 & -l_{1} & -1 & -l_{2} & 0 & 0
\end{array}\right]
$$

\section{B. ODE Formulation for a Three-Body Hinge-Barge Device}

The ODE formulation now is applied to obtain the equations of motion of a three-body hinge-barge device. The vector of independent velocities of the device is:

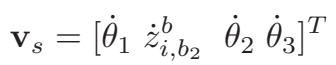

Given the matrix $\mathbf{C}_{z}$ from equation (64), the transformation matrix $\mathbf{P}$ in equation (28) is used to express the relation 


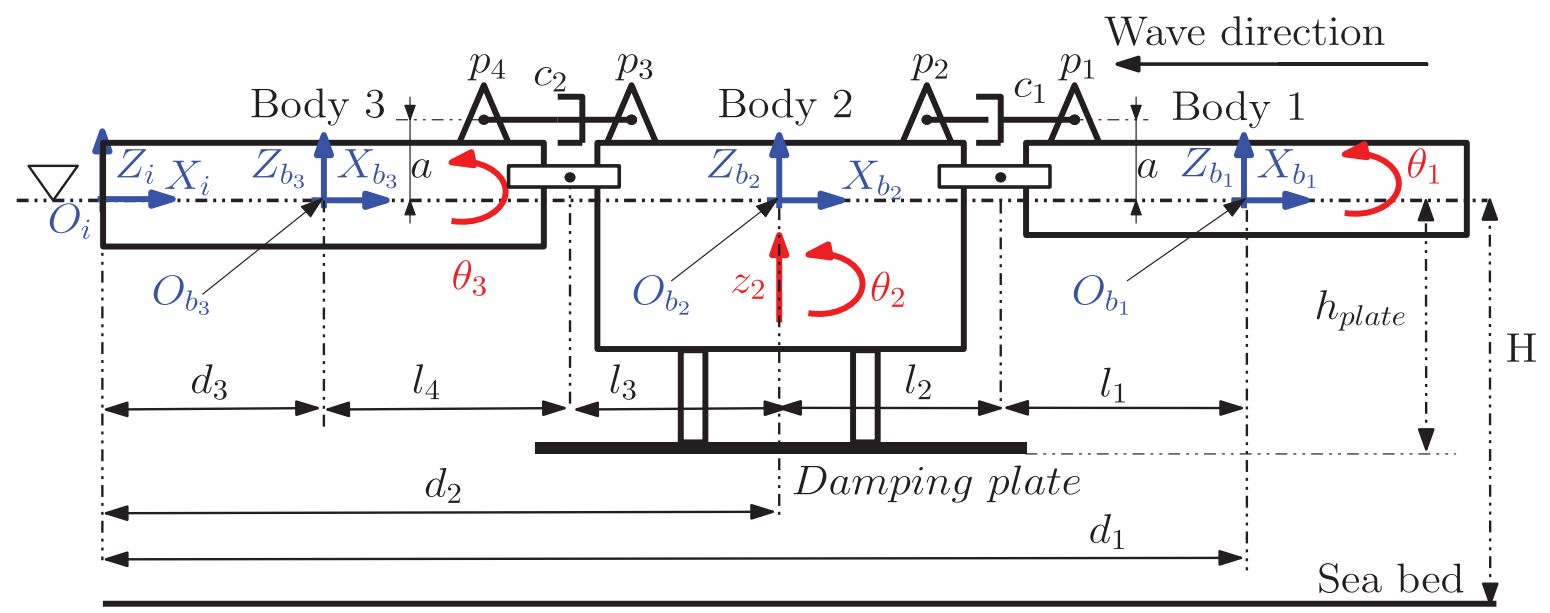

Fig. 2. Three-body hinge-barge device, where $X_{g} Z_{g}$ represents the global frame, and a local frame is assigned to each body composing the device.

between the vector of generalized velocities and independent velocities as follows:

$$
\mathbf{P}=\left[\begin{array}{cccc}
l_{1} & 1 & l_{2} & 0 \\
1 & 0 & 0 & 0 \\
0 & 1 & 0 & 0 \\
0 & 0 & 1 & 0 \\
0 & 1 & -l_{3} & -l_{4} \\
0 & 0 & 0 & 1
\end{array}\right]
$$

Using $\mathbf{P}$, the quantities defined in equations (32)-(39) can be calculated in order to obtain the equations of motion of the device expressed with respect to the degrees of freedom.

\section{Model VALidation}

A specific three-body hinge-barge device was tested in a wave tank using facilities of the U.S. Naval Academy, Annapolis [22]. A tank test with an incident wave made using a Bretschneider spectrum with a significant wave height $H_{s}=15$ $\mathrm{cm}$ and significant period $T=1.276$ seconds was performed, with the direction of the waves along the longitudinal direction of the device. The heave displacement of body 2 and the pitch angles of bodies 1, 2 and 3 were recorded. Given the frequency domain model of the device, the viscous damping matrix $\mathbf{B}_{v i s c, s}$ and the phases of the vector of the excitation

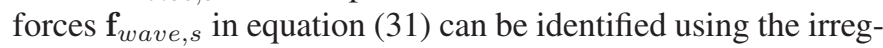
ular wave test. The transfer function in the frequency domain between the degrees of freedom of the device and the incident wave is:

$$
\mathbf{H}(\omega)=\frac{\hat{\mathbf{X}}(\omega)}{\hat{\eta}(\omega)}
$$

where $\hat{\mathbf{X}}(\omega)$ is a complex vector representing the displacements of the degrees of freedom of the device. The transfer function $\mathbf{H}(\omega)$ can be determined experimentally from the tank data [23]. The theoretical transfer function between the degrees of freedom of the device and the incident wave is:

$\hat{\mathbf{H}}(\omega)=\frac{\left|\mathbf{f}_{\text {wave }, s}(\omega)\right| e^{j \angle \mathbf{f}_{\text {wave }, s}(\omega)}}{-\omega^{2}\left(\mathbf{M}_{s}+\mathbf{M}_{\infty, s}\right)+j \omega\left(\mathbf{B}_{\text {visc }, s}+\mathbf{B}_{\text {rad }}(\omega)\right)+\mathbf{G}_{s}}$
The problem of identification of the viscous damping matrix $\mathbf{B}_{v i s c, s}$ and phases of the excitation forces $\angle \mathbf{f}_{\text {wave,s }}(\omega)$ in equation (68) is formulated as an optimization problem as follows:

$$
\min _{\mathbf{B}_{v i s c, s}, \angle \mathbf{f}_{\text {wave }, s}(\omega)} J_{L S}
$$

where the cost function $J_{L S}$ to be minimized is the total sum of squared errors between the transfer functions $\mathbf{H}(\omega)$ and $\hat{\mathbf{H}}(\omega)$, across the range of frequencies of the spectrum of the incident wave,

$$
J_{L S}=\sum_{i=1}^{n_{f}} \sum_{j=1}^{p}\left|H_{j}\left(\omega_{i}\right)-\hat{H}_{j}\left(\omega_{i}\right)\right|^{2}
$$

where $n_{f}$ is the number of frequencies of the spectrum of the incident wave, $p$ is the number of degrees of freedom, $H_{j}$ and $\hat{H}_{j}$ are the experimental and theoretical transfer function between the $j$-th degree of the freedom and the incident wave, respectively. The elements of the viscous damping matrix $\mathbf{B}_{v i s c, s}$ and the phases of the excitation forces $\angle \mathbf{f}_{\text {wave,s }}(\omega)$ at each frequency of the spectrum of the incident wave are used as decision variables for the computation of the minimum of the cost function defined in equation (70).

To validate the identified model, a series of tank tests with an incident wave made using a Jonswap spectrum with a significant wave height $H_{s}=15 \mathrm{~cm}$ and significant period $T=$ 1.276 seconds was performed. PS methods were applied to compute an approximate solution for the equations of motion obtained for the DAE and ODE formulations. Given the linear model of the device derived in section IV, the range of frequencies for the position and velocity spectra is the same as the range of frequencies of the spectrum of the incident wave. Therefore, for a fundamental frequency $\omega_{0}=0.12 \mathrm{rad} / \mathrm{s}$, the order of expansion $N_{x}$ for the position and velocity of the states was 70. Simulations of the device were carried out in Matlab running on a PC with a $3.40 \mathrm{GHz}$ quad core processor and 8 GB RAM. In Figure 3, the frequency response of the heave of body 2 obtained from the tank experiments is compared against the response obtained from the ODE formulation. 


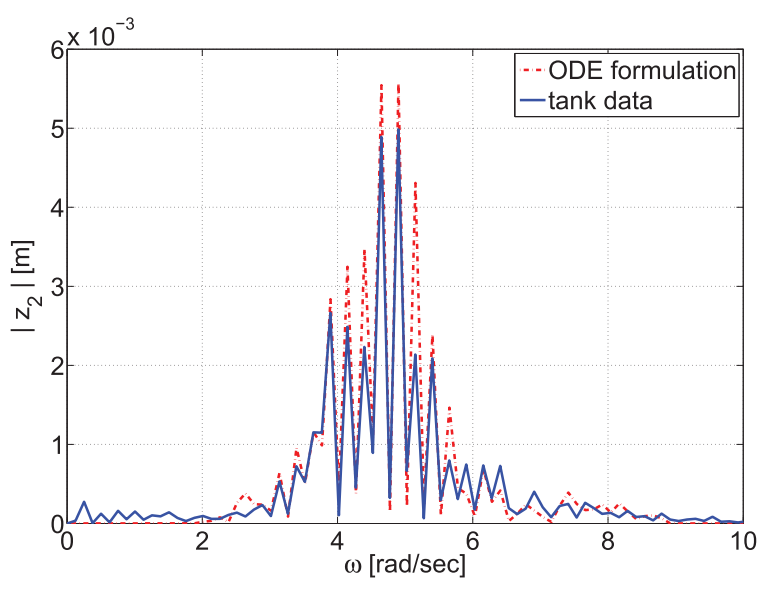

Fig. 3. Frequency response of the heave of body 2 obtained from tank experiments and ODE formulation for an irregular wave made using using Jonswap spectrum with a significant wave height $H_{s}=15 \mathrm{~cm}$ and significant period $T=1.276 \mathrm{sec}$.
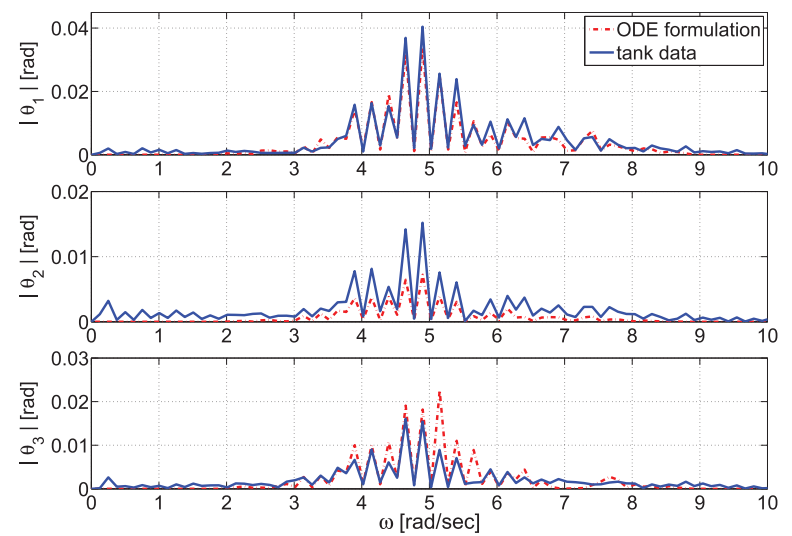

Fig. 4. Frequency response of the pitch angles of body 1, 2 and 3 obtained from tank experiments and ODE formulation for an irregular wave made using using Jonswap spectrum with a significant wave height $H_{s}=15 \mathrm{~cm}$ and significant period $T=1.276 \mathrm{sec}$.

In Figure 4, the frequency responses of the pitch angles of bodies 1,2 and 3 obtained from the tank experiments are compared against the responses obtained from the ODE formulation. In Figure 5, the time domain response of the heave of body 2 obtained from the tank experiments is compared against the response obtained from the ODE formulation. In Figure 6, the time domain responses of the pitch angles of bodies 1,2 and 3 obtained from the tank experiments are compared against the responses obtained from the ODE formulation. Note that the responses of the heave of body 2 and of the pitch angles of the three bodies obtained with the ODE and DAE formulations are identical.

\section{DISCUSSION}

A comparison between the DAE and ODE formulations, and a method based on the integration of the equations of motion, using an explicit Runge-Kutta method (4th order) with a fixed time step, is made. While the Runge-Kutta (R-K) method is used to solve both the transient and steady-state response of the device, the DAE and ODE formulations are used to obtain

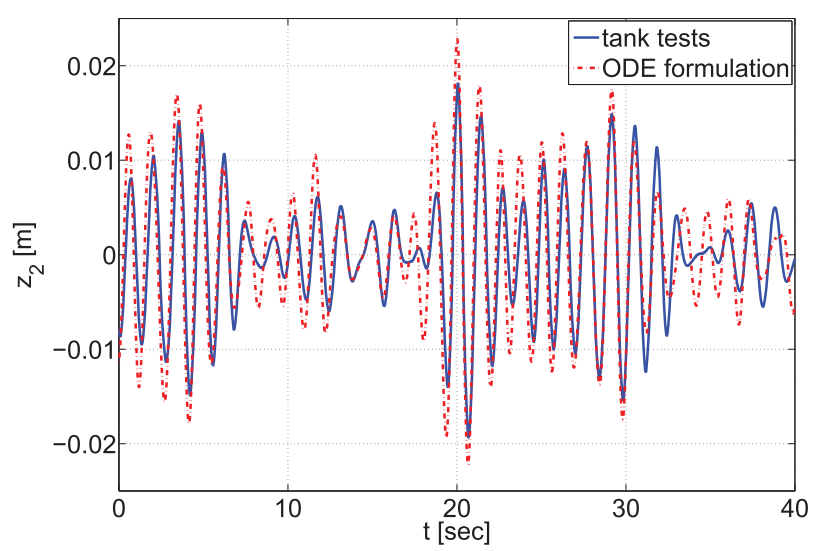

Fig. 5. time domain response of the heave of body 2 obtained from tank experiments and ODE formulation for an irregular wave made using using Jonswap spectrum with a significant wave height $H_{s}=15 \mathrm{~cm}$ and significant period $T=1.276 \mathrm{sec}$.
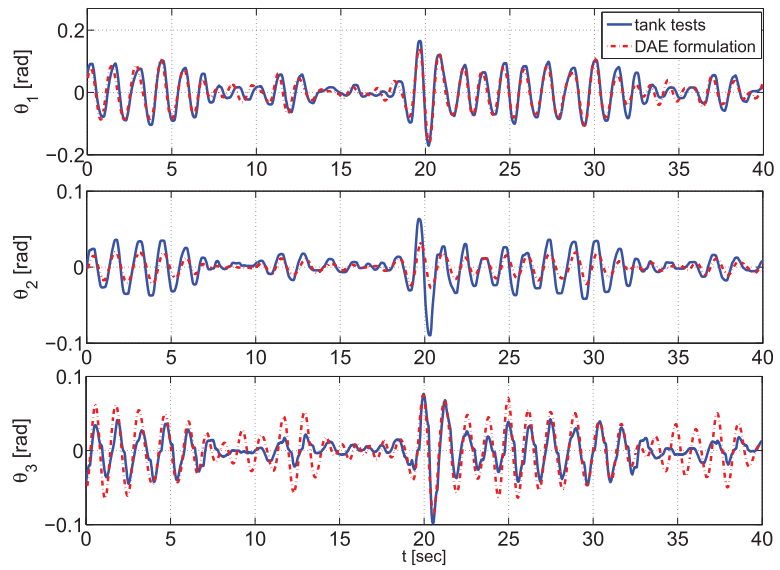

Fig. 6. time domain response of the pitch angles of body 1, 2 and 3 obtained from tank experiments and ODE formulation for an irregular wave made using using Jonswap spectrum with a significant wave height $H_{s}=15 \mathrm{~cm}$ and significant period $T=1.276 \mathrm{sec}$.

the steady-state response of the device only. While there is little overall difference (only for the heave of body 2) between DAE, DOE and R-K methods in reproducing the 3-body validation motion, the small differences between the ODE, DAE and R-K can be explained by the way in which the R-K and PS methods represent the radiation forces. For the R-K method, the convolution integral for the radiation forces is approximated by means of a state-space model [24], while for the PS methods, no approximation is involved [13]. Since the number of frequencies used in the PS methods covers the full range of frequencies of the spectrum of the incident wave, no approximation error is involved.

For a nominal simulation time of 20 s, the computation time $T_{\text {comp }}$ required by the DAE and ODE formulations is compared to that for R-K, for different time step sizes, in Figure 7. Figure 7 confirms that the DAE and ODE formulations are more efficient than R-K for short time steps and, for the DAE and ODE formulations, $T_{\text {comp }}$ does not change significantly with time step, due to the use of a fixed number of collocation points. Note that the DAE formulation is computationally faster than the ODE formulation, for all the time steps. However, it should 


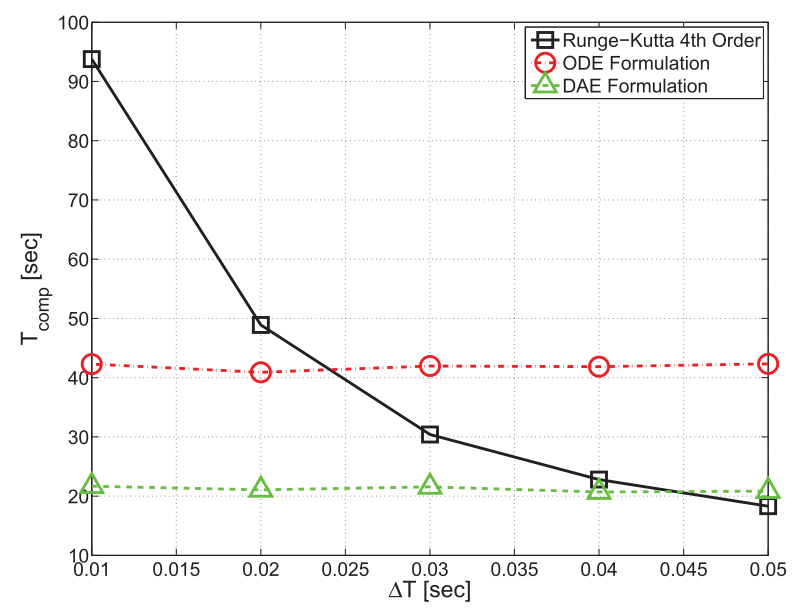

Fig. 7. Computational time required by the Runge-Kutta method, DAE and ODE formulations computed for different time steps and simulation time equal to 20 seconds

be borne in mind that $T_{\text {comp }}$ depends on the order of the finite order radiation damping approximation used in $\mathrm{R}-\mathrm{K}$, with a very small order of 3 used for this baseline comparison. While the size of the time step in the R-K method must be bounded in order to guarantee stability $(\Delta T<0.5 \mathrm{~s})$, PS methods are naturally stable, since motion variables are composed of the sum of bounded functions. For short time steps, the DAE and ODE formulations are computationally faster than R-K. The specific choice of $\Delta T$ depends on the bandwidth of the WEC, $B W_{W E C}$ $\mathrm{Hz}$, with $\Delta T=1 /\left(10 B W_{W E C}\right)$ offered as a guideline.

For a specific time step of $0.02 \mathrm{~s}, T_{\text {comp }}$ for the DAE, ODE and R-K formulations for different simulation times $T_{s}$ is compared in Figure 8, showing that the DAE formulation is more efficient than both the ODE and R-K methods, for all $T_{s}$. The Runge-Kutta method is computationally faster than the ODE formulation for long $T_{s}$, though a higher-order convolution approximation will increase the slope of the R-K trace in Figure 8. Note that, for $T_{s}>50 \mathrm{~s}$, the R-K method is more efficient than the DAE formulation, for the conditions chosen. In terms of likely simulation requirements, system simulation is likely to require a $T_{s}>100 \mathrm{~s}$, while predictive control algorithms will likely employ a prediction horizon of $T_{s}<$ 20s [25].

\section{CONCLUSIONS}

This paper demonstrates that PS methods are a compact and numerically efficient formulation for solving the dynamics of multi-body wave energy devices. Experimental tests on a three-body hinge-barge device with polychromatic waves were carried out to validate the use of the PS models. Both DAE and ODE PS formulations showed good agreement with experimental tests in terms of device motion.

While the DAE and ODE formulations provide the same solution for the motion of the device, the DAE formulation generally requires shorter computational times. With respect to the reference R-K method, PS methods are computationally more stable and require less computational effort for small time steps.

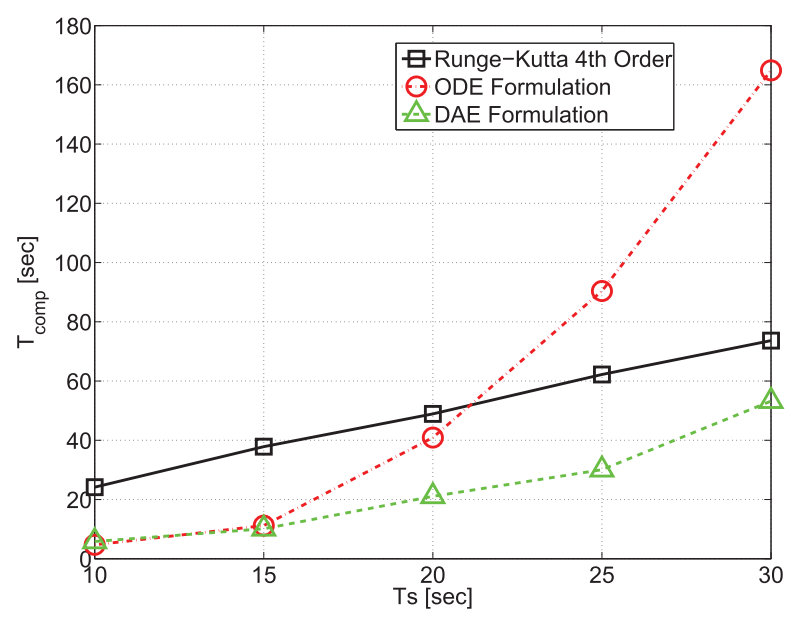

Fig. 8. Computational time required by the Runge-Kutta method, DAE and ODE formulations computed for different simulation times and time step equal to 0.02 seconds

In terms of solution accuracy, PS methods compute an approximation of the solution based on a finite number of expansion coefficients. However, within the bounds of this approximation, the convolution integral corresponding to the WEC radiation damping term is solved exactly. This is in contrast to the R-K method, where the convolution integral is approximated as the output of a finite order system.

However, for the optimal control of multi-body wave energy converters, PS methods are generally more efficient than traditional methods such as Model Predictive Control (MPC), which relies on the simulation capabilities of the integration method in order to compute the optimal solution of the control input. A useful feature of PS is that only a small extra computational effort is required to compute the expansion coefficients for the optimal control force, in addition to the expansion coefficients for the state variables.

\section{REFERENCES}

[1] C. W. Gear, B. Leimkuhler, and G. K. Gupta, "Automatic integration of Euler-Lagrange equations with constraints," J. Comput. Appl. Math., vols. 12-13, pp. 77-90, 1985.

[2] G. A. Maggi, Principii della Teoria Matematica del Movimento Dei Corpi: Corso di Meccanica Razionale. Milano, Italy: Ulrico Hoepli, 1896.

[3] A. Laulusa and O. A. Bauchau, "Review of classical approaches for constraint enforcement in multibody systems," J. Comput. Nonlinear Dyn., vol. 3, no. 1, p. 011004, Jan. 2008.

[4] J. I. Neimark and N. A. Fufaev, Dynamics of Nonholonomic Systems. Providence, RI, USA: AMS, 1972.

[5] H. Hemami and F. C. Weimer, "Modeling of nonholonomic dynamic systems with applications," ASME J. Appl. Mech., vol. 48, pp. 177-182, 1981.

[6] F. E. Udwadia, R. E. Kalaba, and H. C. Eun, "Equations of motion for constrained mechanical systems and the extended D'Alembert's principle," Quart. Appl. Math., vol. 55, no. 2, pp. 321-331, 1997.

[7] J. García de Jalón, J. Unda, A. Avello, and J. M. Jiménez, "Dynamic analysis of three-dimensional mechanisms in "natural" coordinates," ASME J. Mech. Transmiss. Autom. Des., vol. 109, pp. 460-465, 1987.

[8] J. Unda, J. J. García de Jalón, F. Losantos, and R. Enparantza, "A comparative study on some different formulations of the dynamic equations of constrained mechanical systems," ASME J. Mech. Transmiss. Autom. Des., vol. 109, pp. 466-474, 1987.

[9] N. Orlandea, M. A. Chace, and D. A. Calahan, "A sparsity-oriented approach to the dynamic analysis and design of mechanical systemsPart I," ASME J. Eng. Ind., vol. 99, no. 3, pp. 773-779, 1977. 
[10] N. Orlandea, D. A. Calahan, and M. A. Chace, "A sparsity-oriented approach to the dynamic analysis and design of mechanical systemsPart II," ASME J. Eng. Ind., vol. 99, no. 3, pp. 780-784, 1977.

[11] C. Canuto, Y. Hussaini, A. Quarteroni, and T. Zang, Spectral Methods: Fundamentals in Single Domains. New York, NY, USA: Springer, 2006.

[12] B. Fornberg, A Practical Guide to Pseudospectral Methods. Cambridge, U.K.: Cambridge Univ. Press, 1996.

[13] G. Bacelli and J. V. Ringwood, "Numerical optimal control of wave energy converters," IEEE Trans. Sustain. Energy, vol. 6, no. 2, pp. 294 302, Apr. 2015.

[14] T. I. Fossen, Handbook of Marine Craft Hydrodynamics and Motion Control. Hoboken, NJ, USA: Wiley, 2011.

[15] WAMIT User Manual Version 7.0. Cambridge, MA, USA: Massachusetts Institute of Technology.

[16] M. Ó'Catháin, T. I. Fossen, and B. Leira, "Control-oriented modeling of a 2-body interconnected marine structure," in Proc. IFAC Maneouvring Control Marine Craft (MCMC'06), [CD-ROM]. Lisbon, Portugal, 2006.

[17] A. A. Shabana, Dynamics of Multibody Systems. Cambridge, U.K. Cambridge Univ. Press, 2005.

[18] G. Elnagar, M. A. Kazemi, and M. Razzaghi, "The pseudospectral Legendre method for discretizing optimal control problems," IEEE Trans. Autom. Control, vol. 40, no. 10, pp. 1793-1796, Oct. 1995.

[19] I. M. Ross and M. Karpenko, "A review of pseudospectral optimal control: From theory to flight," Аnnu. Rev. Control, vol. 36, no. 2, pp. 182-197, 2012.

[20] A. Falcão, "Wave energy utilization: A review of the technologies," Renew. Sustain. Energy Rev., vol. 14, no. 3, pp. 899-918, 2010.

[21] Sea Power Ltd. [Online]. Available: http://www.seapower.ie

[22] A. Paulmeno, "An experimental analysis of the optimal design conditions for a model scale McCabe wave pump," Dept. Naval Archit. Ocean Eng., United States Naval Academy Annapolis, Annapolis, MD, USA, Independent Research in Ocean Engineering Honors Final Report, May 2013.

[23] R. Genest, "Développement et validation expérimentale de stratégies de contrôle des récupérateurs de l'énergie des vagues," Ph.D. dissertation, École centrale de Nantes, Nantes, France, 2015.

[24] T. Perez and T. I. Fossen, "A matlab toolbox for parametric identification of Radiation-Force Models of Ships and Offshore Structures," Model. Ident. Control, vol. 30, no. 1, pp. 1-15, 2009.

[25] F. Fusco and J. V. Ringwood, "A study on the prediction requirements in real-time control of wave energy converters," IEEE Trans. Sustain. Energy, vol. 3, no. 1, pp. 176-184, 2012.

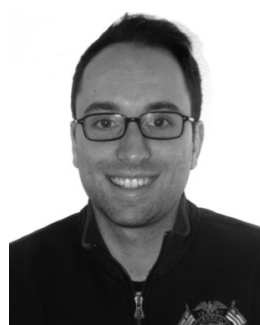

Francesco Paparella received the Laurea Magistrale degree in automation engineering from the Politecnico di Milano, Milano, Italy, in 2012. Since October 2013, he is pursuing the Ph.D. degree at the Centre for Ocean Energy Research, Maynooth University, Maynooth, Ireland. He worked on the load sharing optimization of compression stations with the ABB Corporate Research Center, Baden (Zurich), Switzerland. From January 2013 to September 2013, he worked with RSE S.p.a., Milano, Italy, as a Researcher for the development of

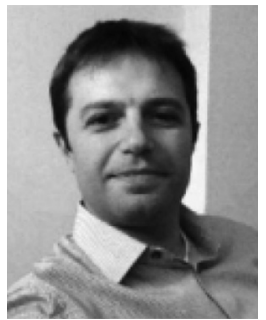

Giorgio Bacelli received the Laurea Magistrale degree in electronic engineering from the Università Politecnica delle Marche, Ancona, Italy, and the $\mathrm{Ph} . \mathrm{D}$. degree in electronic engineering from the Centre for Ocean Energy Research, Maynooth University, Maynooth, Ireland, in 2006 and 2014, respectively. He is currently with Sandia National Laboratories, Albuquerque, NM, USA, working on the design and implementation of control strategies for wave energy converters.

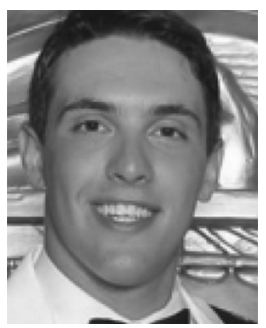

Andrew Paulmeno received the B.S. degree (with Hons.) in ocean engineering from the U.S. Naval Academy, Annapolis, MD, USA, in 2013. He is currently a Combat Engineer Officer on active duty with the United States Marine Corps. His research interests include wave energy converters and conditional design optimization.

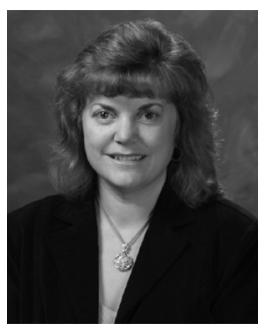

Sarah E. Mouring received the Bachelor's degree in civil engineering from the University of Delaware, Newark, DE, USA, and the M.Sc. and Ph.D. degrees in civil engineering from Johns Hopkins University, Baltimore, MD, USA, in 1987, 1990, and 1993, respectively. Since 1992, she has been a Professor with the Department of Naval Architecture and Ocean Engineering, U.S. Naval Academy, Annapolis, MD, USA, where she is currently the Ocean Engineering Program Director. Her research interests include composite materials and wave energy conversion.

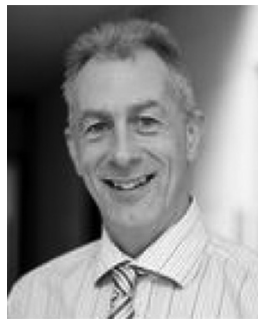

John V. Ringwood (M'87-SM'97) received the Diploma degree in electrical engineering from Dublin Institute of Technology, Dublin, Ireland, and the $\mathrm{Ph} . \mathrm{D}$. degree in control of steel mills from Strathclyde University, Glasgow, U.K., in 1981 and 1985, respectively. He is currently a Professor of Electronic Engineering with Maynooth University, Maynooth, Ireland. He was the Head of the Department of Electronic Engineering, Maynooth University from 2000 to 2006. He is a Chartered Engineer and a Fellow of the Institution of Engineers of Ireland. His research interests include time series modeling, wave energy, control of plasma processes, and biomedical engineering.

electrical systems. 